\title{
Inflammation-associated suppression of metabolic gene networks in acute and chronic liver disease
}

\author{
Gisela Campos ${ }^{1}$. Wolfgang Schmidt-Heck ${ }^{2}$ Jonathan De Smedt ${ }^{3} \cdot$ Agata Widera $^{1} \cdot$ Ahmed Ghallab $^{1,4}$. \\ Larissa Pütter ${ }^{1}$. Daniela González ${ }^{1} \cdot$ Karolina Edlund $^{1}$. Cristina Cadenas ${ }^{1} \cdot$ Rosemarie Marchan $^{1} \cdot$ Reinhard Guthke $^{2}$. \\ Catherine Verfaillie $^{3}$. Claudio Hetz ${ }^{5,6,7,8} \cdot$ Agapios Sachinidis $^{9} \cdot$ Albert Braeuning $^{10,11}$ - Michael Schwarz ${ }^{10}$. \\ Thomas S. Weiß ${ }^{12}$ - Benjamin K. Banhart ${ }^{13}$. Jan Hoek ${ }^{13}$. Rajanikanth Vadigepalli ${ }^{13}$. Jeffrey Willy ${ }^{14}$. \\ James L. Stevens ${ }^{15}$. David C. Hay ${ }^{16}$. Jan G. Hengstler ${ }^{1} \cdot$ Patricio Godoy ${ }^{1}$
}

Received: 13 August 2019 / Accepted: 20 November 2019 / Published online: 9 January 2020

(c) Springer-Verlag GmbH Germany, part of Springer Nature 2020

\begin{abstract}
Inflammation has been recognized as essential for restorative regeneration. Here, we analyzed the sequential processes during onset of liver injury and subsequent regeneration based on time-resolved transcriptional regulatory networks (TRNs) to understand the relationship between inflammation, mature organ function, and regeneration. Genome-wide expression and TRN analysis were performed time dependently in mouse liver after acute injury by $\mathrm{CCl}_{4}(2 \mathrm{~h}, 8 \mathrm{~h}, 1,2,4,6,8,16$ days), as well as lipopolysaccharide (LPS, $24 \mathrm{~h}$ ) and compared to publicly available data after tunicamycin exposure (mouse, $6 \mathrm{~h}$ ), hepatocellular carcinoma (HCC, mouse), and human chronic liver disease (non-alcoholic fatty liver, HBV infection and HCC). Spatiotemporal investigation differentiated lobular zones for signaling and transcription factor expression. Acute $\mathrm{CCl}_{4}$ intoxication induced expression of gene clusters enriched for inflammation and stress signaling that peaked between 2 and $24 \mathrm{~h}$, accompanied by a decrease of mature liver functions, particularly metabolic genes. Metabolism decreased not only in pericentral hepatocytes that underwent $\mathrm{CCl}_{4}$-induced necrosis, but extended to the surviving periportal hepatocytes. Proliferation and tissue restorative TRNs occurred only later reaching a maximum at $48 \mathrm{~h}$. The same upstream regulators (e.g. inhibited RXR function) were implicated in increased inflammation and suppressed metabolism. The concomitant inflammation/metabolism TRN occurred similarly after acute LPS and tunicamycin challenges, in chronic mouse models and also in human liver diseases. Downregulation of metabolic genes occurs concomitantly to induce inflammation-associated genes as an early response and appears to be initiated by similar upstream regulators in acute and chronic liver diseases in humans and mice. In the acute setting, proliferation and restorative regeneration associated TRNs peak only later when metabolism is already suppressed.
\end{abstract}

Keywords Liver injury $\cdot$ Regeneration $\cdot$ Transcriptomics $\cdot$ Gene networks

\section{Abbreviations}

HCC Hepatocellular carcinoma

NAFLD Non-alcoholic fatty acid liver disease

Gisela Campos and Wolfgang Schmidt-Heck equal contribution.

Electronic supplementary material The online version of this article (https://doi.org/10.1007/s00204-019-02630-3) contains supplementary material, which is available to authorized users.

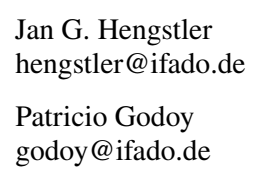

Extended author information available on the last page of the article
TRN Transcriptional regulatory networks

IPA Ingenuity pathway analysis

Tm Tunicamycin

LPS Lipopolysaccharide

HBV Hepatitis virus type B

\section{Introduction}

Inflammation serves to initiate tissue repair by elimination of the causes of injury such as infectious agents and necrotic cells (Karin and Clevers 2016; Campos et al. 2014). In recent years, computational modeling of transcriptional regulatory networks (TRNs) as regulatory interactions, among 
transcription factors and their target genes have been used to gain insight into genome-wide expression patterns associated with inflammation and have established a link between inflammatory and regenerative responses (Karin and Clevers 2016; Michalopoulos 2013). However, a time-resolved genome-wide analysis of acute inflammation of the liver and a comparison to chronic liver diseases in mouse and human has not yet been performed. To gain deeper insight, we generated time-resolved genome-wide expression data using the well-established protocol of acute injury and inflammation induced by $\mathrm{CCl}_{4}$ in mouse liver. TRNs obtained in this mouse model were compared to chronic liver inflammation in mouse and human liver tissue, including NAFLD, cirrhosis, HBV infection, and HCC. We report a common feature in all acute and chronic liver conditions, namely upregulation of inflammation-associated genes that is accompanied by strong downregulation of a large set of genes responsible for mature parenchymal functions, predominantly metabolism. Inflammation-associated downregulation of mature tissue functions is mediated by a similar set of upstream regulators in mouse and human.

\section{Materials and methods}

A detailed description of all methods, including data processing and visualization, fuzzy clustering and gene set enrichment analysis, ingenuity pathway analysis, immunostaining and microscopy, western blot, RNA isolation, cDNA synthesis, real-time quantitative PCR, and mouse experiments are described in Supplement 1. Briefly, differentially expressed genes in mouse liver after acute $\mathrm{CCl}_{4}$ intoxication with $1.6 \mathrm{~g} / \mathrm{kg}$ (intraperitoneal) were determined using Affymetrix A450 2.0 gene chips, (Array Express E-MTAB-24445). Liver tissue specimens were collected from five C57BL6/N male mice $2 \mathrm{~h}, 8 \mathrm{~h}$, day $1,2,4,6$, 8 , and 16 after $\mathrm{CCl}_{4}$ intoxication. Control liver tissue was obtained from mice on day 1 after receiving vehicle (olive oil) intraperitoneal injections. Inflammation-dependent differentially expressed genes were identified by analysis of mouse liver tissue $24 \mathrm{~h}$ after intraperitoneal administration of lipopolysaccharide $(750 \mathrm{ng} / \mathrm{kg})$. Moreover, the following expression data sets were used: mouse liver tissue $6 \mathrm{~h}$ after intraperitoneal administration of tunicamycin $(1 \mathrm{mg} / \mathrm{kg})$ (GSE29929); pericentral (PC) and periportal (PP) enriched hepatocytes; hepatocellular carcinoma (GSE30485); gene array data from human liver tissue: non-alcoholic fatty liver disease (NAFLD, score 0-1, 3-4) (GSE49541); liver tissue infected with hepatitis B (GSE14668); liver cirrhosis and human hepatocellular carcinoma (GSE17548). An overview of the expression data used in the present study is given in Fig. 1. Tables containing differentially expressed genes and biostatistical raw data of all data sets are available in
Supplements 2-17. Legends giving an overview over all Supplements are given in Supplement 1.

Clusters of correlated genes based on similar timedependent fold change after $\mathrm{CCl}_{4}$ intoxication were generated by fuzzy c-means clustering with the fuzziness $m=2$. To cluster the gene expression profiles of DEGs, their profiles were logarithmized and scaled between the values -1 and 1. The optimal number of clusters was estimated using the 'Separation index'. A gene is assigned to the cluster with the highest degree of membership and if the maximum degree of membership is less than 0.3 , the gene is regarded as unassignable and assigned to the group of outliers. A list with the genes contained in each cluster and the respective expression change can be found in the supplemental section.

\section{Results}

\section{Inflammation-associated suppression of mature liver functions}

To understand the TRNs activated in acute liver injury, we performed a time-resolved Affymetrix gene array study on mouse liver after acute intraperitoneal administration of $\mathrm{CCl}_{4}$ (1.6 g/kg in olive oil) (Campos et al. 2014) (Fig. 1a, b; Suppl. Fig. 1 in Supplement 1). In total, 3750 differentially expressed genes were identified (1.5 fold, $p<0.05$; FDR adjusted) (Supplement 2). Principal component analysis (PCA) showed clear differences to controls and good clustering of time-matched liver tissue replicates at $2 \mathrm{~h}, 8 \mathrm{~h}$, and on days 1, 2, and 4 after $\mathrm{CCl}_{4}$ administration; at days 6, 8, and 16, expression data were similar to controls again (Fig. 1b). The high reproducibility between replicates offered good conditions for a time-resolved bioinformatics analysis. Consequently, the fuzzy c-means algorithm was applied, which revealed eight time-dependent gene clusters, including 3655 genes (Fig. 2) (Supplement 3).

Overrepresented gene ontology (GO) and KEGG pathway annotations were used to identify biological motifs associated with time-dependent progression of transcriptional alterations (Fig. 2; Supplement 4). Because similar genes and enriched $\mathrm{GO} / \mathrm{KEGG}$ terms were present in some of the eight gene clusters, we joined them into three cluster groups. Cluster group 1 included upregulated genes that peaked between 2 and 24 h (Fig. 2, clusters 1a-d). This cluster group contained genes associated with inflammation, cell stress, and the unfolded protein response (UPR). The earliest induced genes (maximal expression at $2 \mathrm{~h}$; cluster 1a) were enriched for the MAPK and TNF pathways, while subsequent clusters were mostly enriched in inflammation and ER stress/proteostasis associated genes. Cluster group 1 included multiple intracellular signaling components and transcription factors (e.g. Gadd45g, Jun, Atf4, Maff) as well 
(A)

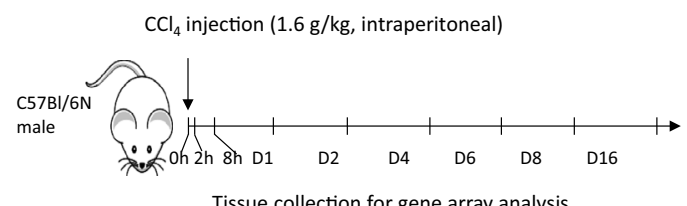

(B)

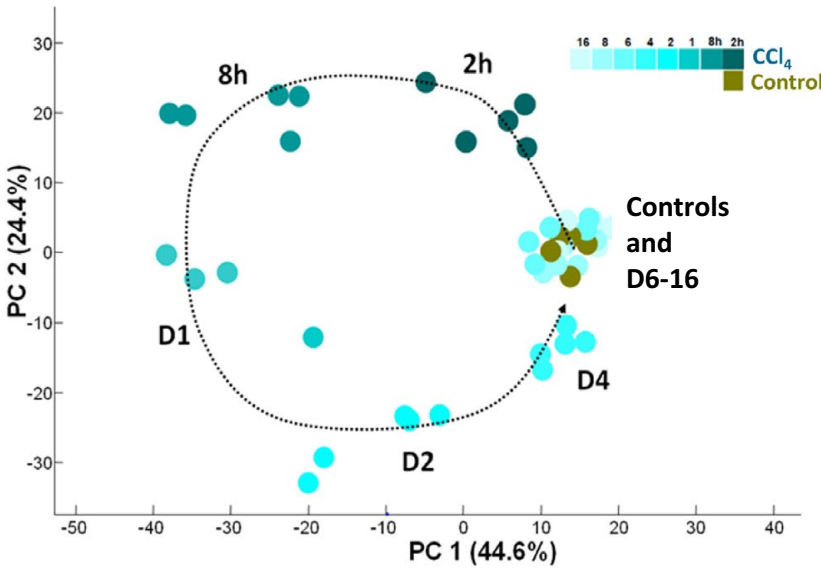

(C)

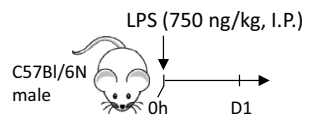

(D)

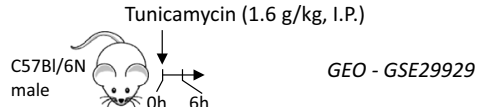

(E)

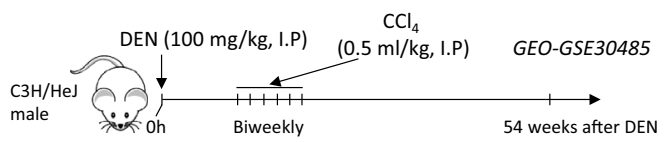

\section{(F)}

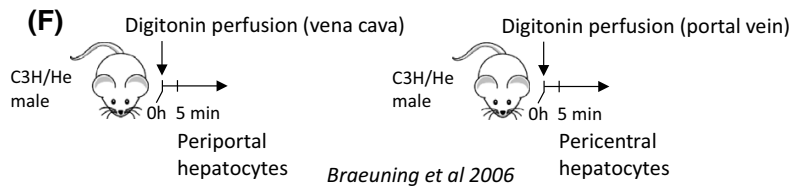

(G)

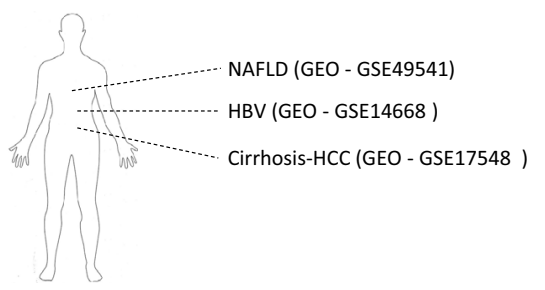

Fig. 1 Experimental design, principal component and cluster analysis of time-resolved expression data after $\mathrm{CCl}_{4}$ intoxication. a Overview of the experimental setup for acute intoxication with $\mathrm{CCl}_{4}$. Liver tissue sections were collected at the indicated time points after a single intraperitoneal injection of $\mathrm{CCl}_{4}(1.6 \mathrm{~g} / \mathrm{kg}) . N=5$ mice per time point. b Principal component analysis of the top 1000 differentially expressed genes in the combined time series after $\mathrm{CCl}_{4}$. The different

as growth factors and cytokines (e.g. Fgf21, Cxcl1, Ccl2, Ccl6) (Fig. 2).

Cluster group 2 included downregulated genes that reached a minimum of expression at 8 and $24 \mathrm{~h}$ upon $\mathrm{CCl}_{4}$ administration, thereby responding in the same time interval as the upregulated genes of cluster group 1 (Fig. 2; clusters $2 \mathrm{a}, \mathrm{b})$. Cluster group 2 was enriched in genes associated with mature liver function, particularly metabolism; it included the GO annotations "Small molecule metabolic process", "Organic acid metabolic process", and the KEGG pathways "Valine, leucine and isoleucine degradation", "Steroid hormone biosynthesis" and "Drug metabolism-cytochrome P450". Selected individual genes from the different cluster groups were validated by qRT-PCR (Suppl. Fig. 2 in Supplement 1). Together, cluster groups 1 and 2 showed a time-resolved transient induction of genes associated with stress signaling, inflammation, and ER stress that occurred simultaneously with a massive downregulation of genes associated with metabolism and further functions of the mature liver.

Cluster group 3 genes responded later than the genes of cluster groups 1 and 2, showing maximal expression not blue tones denote the time points of each biological replica (mouse) for liver tissue after $\mathrm{CCl}_{4}$ administration. Control liver tissues (day 1 treated with olive oil) are indicated in dark green. $\mathbf{c}-\mathbf{f}$ Published genome-wide data from mouse models used for comparison in the present study. g Genome-wide data from human liver tissue used for comparison with the mouse data (color figure online)

before day 2 after $\mathrm{CCl}_{4}$ intoxication (Fig. 2, clusters 3a, b). These clusters represent genes associated with proliferation, wound healing, and immune cell infiltration. They correspond to GO terms such as "Cell cycle", "ECM organization" and "Immune response", and the KEGG terms "DNA replication".

\section{Identification of TRNs in acute liver injury and regeneration}

Having identified the most significant biological motifs activated during liver injury and regeneration, we sought to establish the TRNs controlling the aforementioned time-dependent gene clusters. KEGG pathway enrichment analysis provided only little insight into the critical TRNs controlling the deregulated genes. Therefore, a more accurate representation of TRNs was established by identifying (1) deregulated transcription factors, (2) overrepresented transcription factor binding sites (TFBS) in the promoter/ enhancers (Supplement 4), and (3) upstream regulator inference using ingenuity pathway analysis (IPA) (Supplement 5). These approaches revealed tremendous details of TRNs 


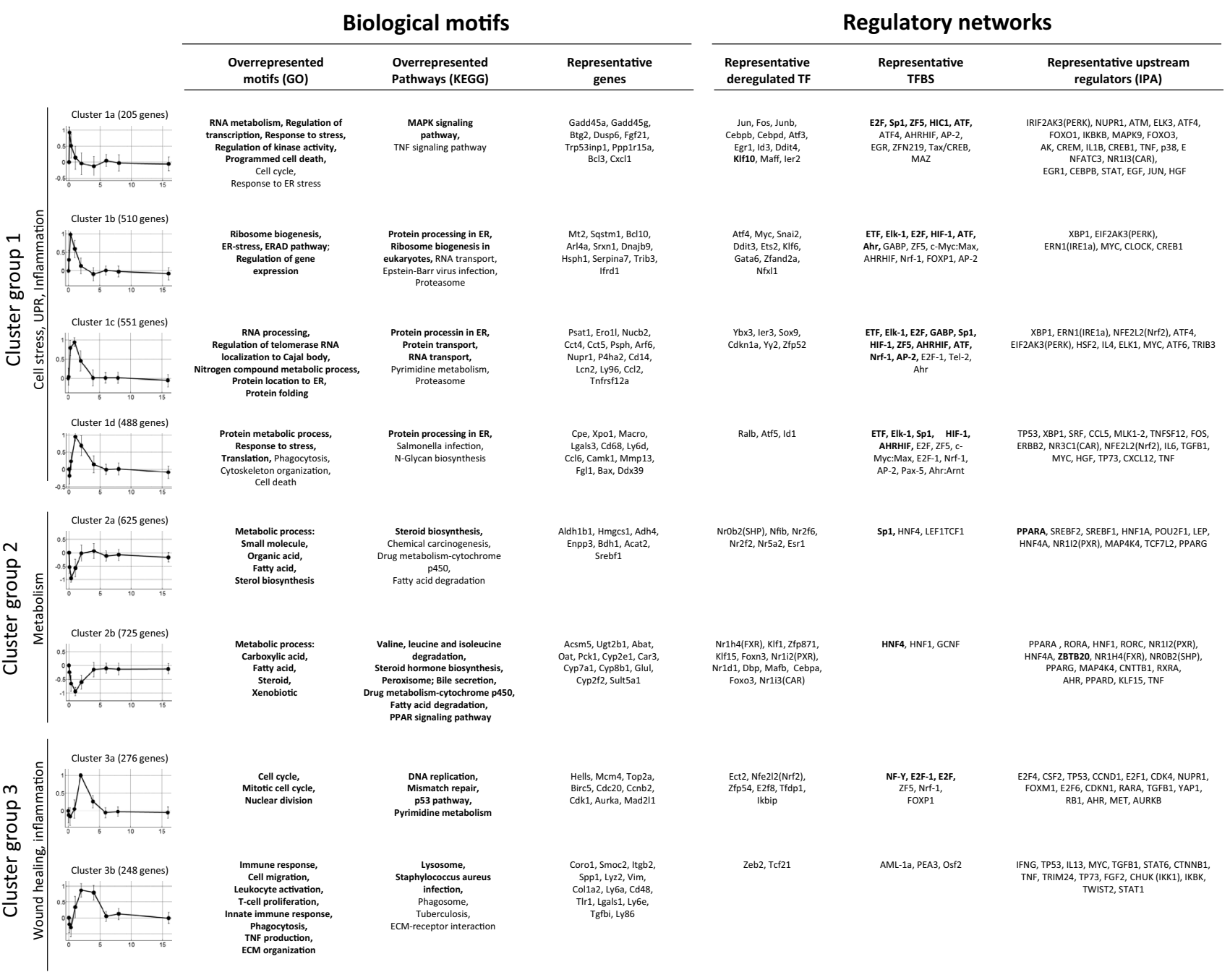

Fig. 2 Grouping of time-resolved gene clusters in mouse liver after $\mathrm{CCl}_{4}$ intoxication. Time-dependent fuzzy clustering was performed with deregulated genes in mouse liver tissue after $\mathrm{CCl}_{4}$ intoxication. Dots represent mean scaled values of all genes in each cluster. Error bars represent standard deviations. The three cluster groups were formed based on similarities in overrepresented gene ontology (GO), KEGG motifs, and pathways identified by ingenuity pathway

components in each cluster group (Fig. 2). For example, TFs upregulated in cluster group 1 included well-known stress regulators, such as Fos, Jun, Cebpb, and Egr1, as well as TFs that have not yet been associated with acute liver injury, including Klf10, Klf6, Ybx3, and Sox9. Cluster group 2 contained downregulated TFs with known roles in metabolic functions of healthy liver such as $\mathrm{NrOb} 2$ (SHP), Nr1h4 (FXR), Nr112 (PXR) and Cebpa and TFs with no known function in liver metabolism, for example Esr1, Zfp871, and Nr1d1 (Supplement 5).

TFBS enrichment and IPA analysis provided additional information on transcriptional regulators whose expression is not transcriptionally altered during acute liver injury. For example, TFBS overrepresented in cluster groups 1 analysis (IPA). The figure shows the most significantly enriched GO and KEGG annotations and representative deregulated genes for each cluster. Representative transcription factors (TF) include the strongest deregulated genes with known function as transcriptional regulator identified in each cluster. The representative transcription factor binding sites and representative pathways (IPA) correspond to those with highest statistical significance $\left(p<10^{-5}\right)$

included HIF-1, AHR/HIF, Foxp1, ETF, and Elk-1 (Fig. 2; Supplement 5). Cluster group 2 was enriched in HNF4 and HNF1 TFBS. Further TRN modulators were identified in cluster groups 1 by IPA, including ER stress signaling members Xbp1 (activated by splicing), Eif2AK3 (PERK), and ERN1 (IRE1a) (activated by phosphorylation), Ikbkb and Nfe212 (activated by proteolytic cleavage) and $\mathrm{Nr} 113$ (CAR) (activated by ligand engagement) in (Fig. 2; Supplement 5). In cluster group 2, IPA revealed well-known regulators of liver metabolism (e.g. HNF4, PPARA, SREBF1 and 2, and PPARG) and regulators associated with upregulated gene clusters such as TNF and MAP4K4 (Fig. 2; Supplement 5). 


\section{Characterization of spatiotemporal signaling during $\mathrm{CCl}_{4}$-induced liver injury}

To investigate the spatiotemporal relationships of signaling in liver parenchyma, immunohistochemical analysis of transcription factors in liver sections of $\mathrm{CCl}_{4}$-treated mice were performed. First, we focused on cluster group 1 factors c-Jun and CEBPD. c-Jun expression was not detected in control tissues, but increased in hepatocyte nuclei, particularly, in PC hepatocytes $2 \mathrm{~h}$ after $\mathrm{CCl}_{4}$ administration (Fig. 3a; Suppl. Fig. 3 in Supplement 1). On day 1, when the dead cell area is clearly discernible, c-Jun staining slightly exceeded the PC dead area, but never reached periportal hepatocytes (Fig. 3a). This is comparable to the PC expression of the ER stress-associated transcription factor $\mathrm{CHOP}$ after $\mathrm{CCl}_{4}$ intoxication (Suppl. Fig. 4 in Supplement 1 and [2]). The inflammation-associated transcription factor CEBPD from cluster group 1A exhibited a different response (Fig. 3a). At $2 \mathrm{~h}$, nuclear staining was already evident in both PC and PP hepatocytes. To assess control mechanisms of cluster group 2 genes, we analyzed expression of HNF4, which showed an inverse expression pattern to the stress and inflammation markers c-Jun and CEBPD. HNF4 was expressed in all hepatocytes of control mice (Fig. 3a). After $8 \mathrm{~h} \mathrm{CCl}_{4}$ treatment, it decreased in the PC region and after $24 \mathrm{~h}$ reached a similarly low level all over the liver lobule. In summary, stress signaling by c-Jun and CHOP occurred predominantly in pericentral hepatocytes, while CEBPD and HNF4, regulatory factors of inflammation and metabolism, were altered in all lobular zones.

The suppression of HNF4 in all lobular zones suggests that metabolic liver functions were not only affected in pericentral hepatocytes, which will undergo necrosis, but also PP hepatocytes. To systematically assess this hypothesis, we analyzed genes from PP- and PC-enriched hepatocytes (Braeuning et al. 2006) (Supplement 6). Venn diagram analysis of the zonated genes (either expressed in PP or in PC hepatocytes) indicated the largest overlaps with clusters $2 \mathrm{a}$ and $2 b$ (Fig. 3b; Supplements 7, 8). Noteworthy, the genes with the highest PP-to-PC ratio were among those overlapping with cluster group 2 genes. $\mathrm{CCl}_{4}$ does not cause cytotoxicity to PP hepatocytes, because in contrast to PC cells, they do not express Cyp2e1, which is required for metabolic activation of $\mathrm{CCl}_{4}$. Therefore, the strong expression alterations in the PP cells may represent an adaptation to $\mathrm{PC}$ hepatocyte death. In contrast to the genes downregulated in response to $\mathrm{CCl}_{4}$, zonal genes minimally overlap with upregulated genes in cluster groups 1-3 (Fig. 3b). In conclusion, spatiotemporal analysis reveals zonated (stress) versus non-zonated (inflammation and metabolism) signaling motifs during acute liver injury.

Since cluster 1a (Fig. 2) suggested increased stress signaling, e.g. via Fos, Jun, STAT and MAPK, western blot analyses were performed. A transient increase in pSTAT3, pAkt, pJNK, and pERK was evident already $2 \mathrm{~h}$ after $\mathrm{CCl}_{4}$ injection (Fig. 3c). A biphasic behavior for pAkt and pERK was observed, with a second peak on day 1 for pAkt and day 2 for pERK (Fig. 3c; quantifications: Suppl. Fig. 3 in Supplement 1). These results correspond to the aforementioned cluster group 1 stress signaling networks. Similarly, the proliferation marker PCNA peaked on days 2 and 4 (Fig. 3c), in agreement with the timing of the wound healing gene networks (cluster group 3). Cell stress is often accompanied by increased autophagy. To assess possible alterations in autophagy during $\mathrm{CCl}_{4}$ intoxication, the autophagosome factors LC3 and p62 were analyzed. Conversion of LC3-I to LC3-II was observed between $2 \mathrm{~h}$ and day 1 after $\mathrm{CCl}_{4}$ administration (Fig. 3d); concurrently the cargo protein p62 and polyubiquitinated proteins accumulated between 2 and $24 \mathrm{~h}$ after $\mathrm{CCl}_{4}$, suggesting an altered autophagic flux.

\section{Inflammation/ER stress and metabolic gene networks are influenced by the same upstream regulators}

When all genes were plotted time dependently, an almost mirror-inverted pattern was obtained for upregulated and downregulated genes up to approximately 4 days after $\mathrm{CCl}_{4}$ administration (Fig. 4a). Downregulation (similar as upregulation) occurred already at the earliest tested time period of $2 \mathrm{~h}$, when pericentral hepatocytes are not yet necrotic. Therefore, decreased gene levels during this time period are unlikely to result from degradation of pericentral liver tissue, but rather are due to an active process. To identify possible transcriptional regulatory motifs controlling the up and downregulation of these genes, we first analyzed individual time points during the early phase of stress and injury ( $2 \mathrm{~h}$ till day 1 ) using all differentially expressed genes at each time point. The top ten pathways detected by IPA were related to inflammation, unfolded protein response, and metabolism (Fig. 4b). The most significantly enriched pathway identified at $8 \mathrm{~h}$ and day 1 was "LPS/IL-1 mediated inhibition of RXR function" (Supplement 9). Similarly, analysis of upstream regulators showed a strong activated score for regulators of inflammation and ER stress, while regulators of liver metabolic functions were estimated as highly inactive (Fig. 4c; Supplement 10).

A key result was that the strongest enriched canonical IPA pathway, "LPS/IL-1 Mediated Inhibition of RXR Function" included both highly upregulated inflammation-associated genes and downregulated genes associated with mature liver functions (Fig. 4d). For example, the inflammationmediating pathway c-Jun was the strongest up and the apical bile salt export carrier BSEP, representing a function of the mature liver, was among the strongest downregulated genes both triggered by the same canonical pathway. Similar 

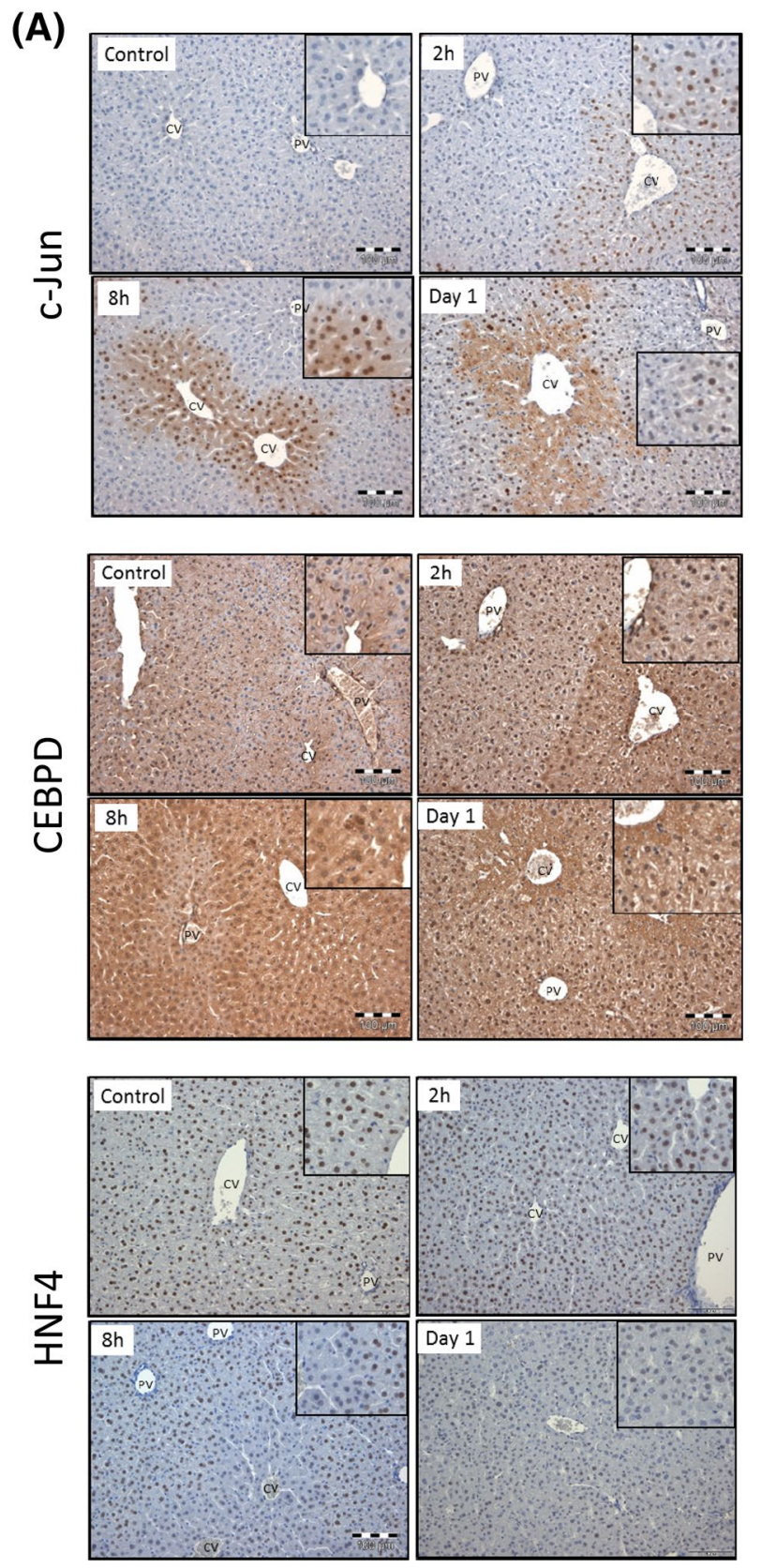

(C)

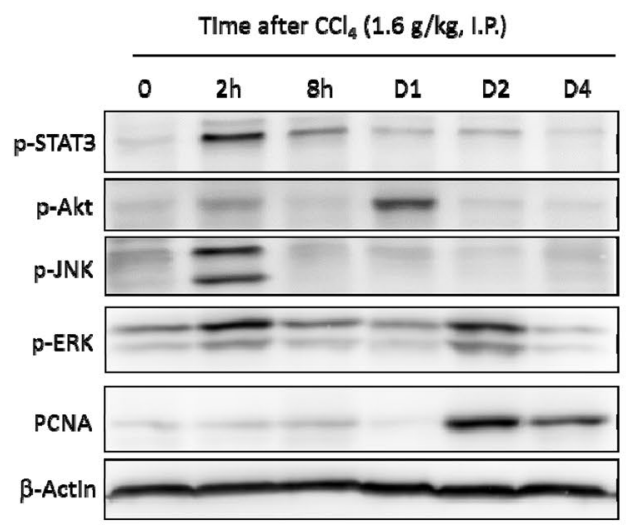

(B)
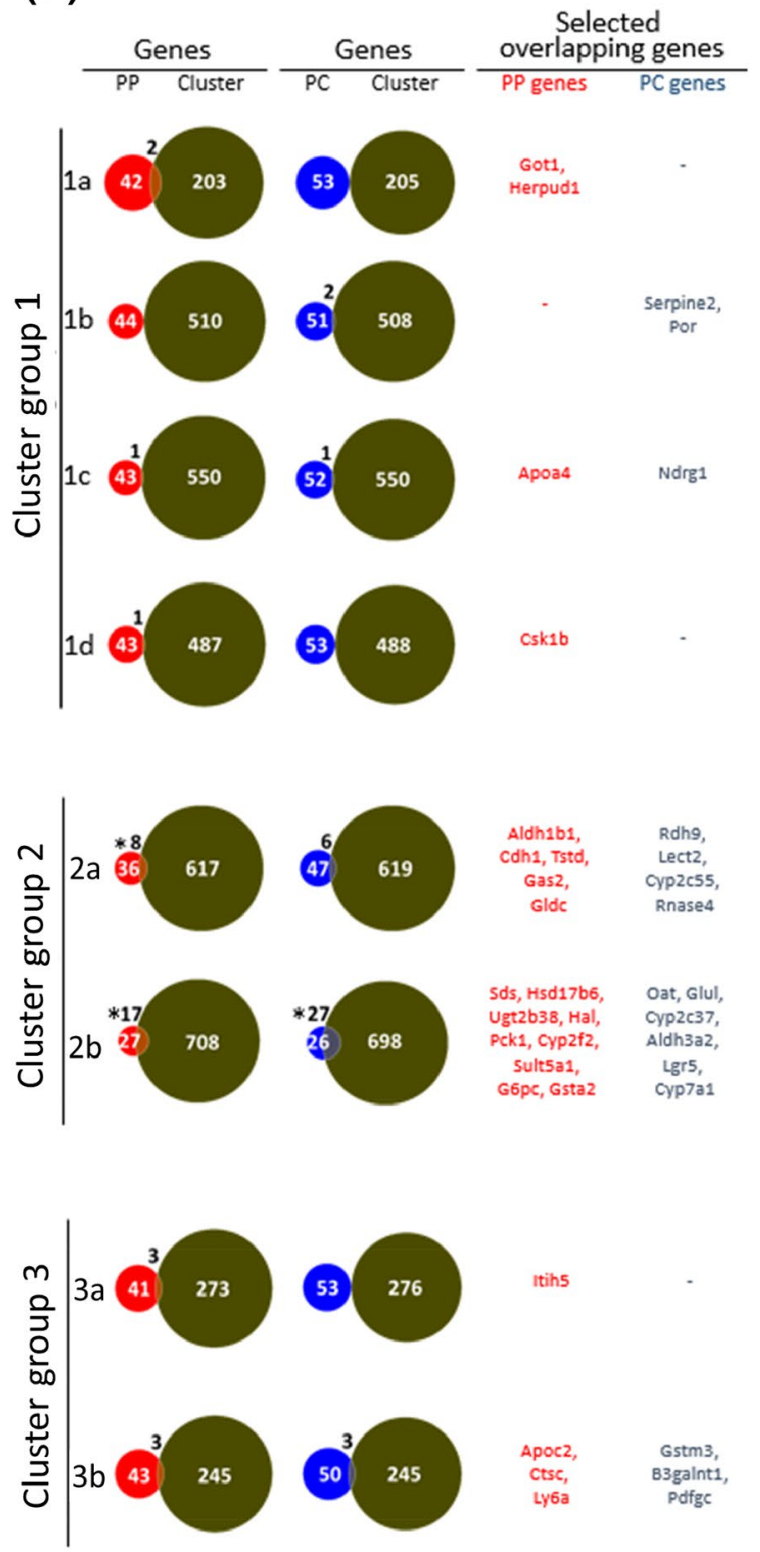

$\left(*=p<10^{-5}\right)$

(D)

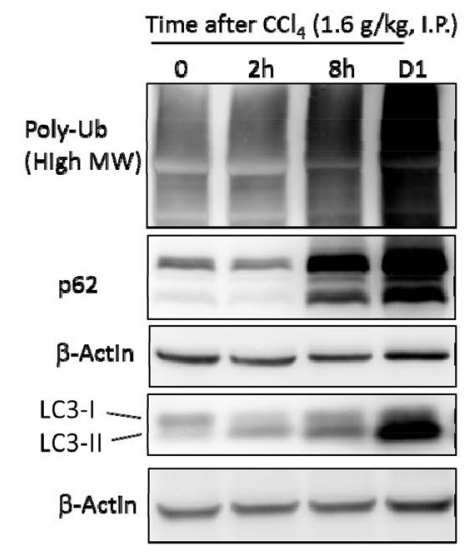


4Fig. 3 Spatiotemporal zonation of signaling and gene expression during $\mathrm{CCl}_{4}$ intoxication. a Immunostaining analysis of c-Jun, CEBPD, and HNF4 during acute liver injury. Control tissues correspond to mouse liver on day 1 after injection of vehicle (olive oil). Representative of at least three biological replicas per time point and condition. b Venn diagrams showing overlap between PP- or PC-enriched genes and the three $\mathrm{CCl}_{4}$ cluster groups. Red and blue circles represent $\mathrm{PP}$ or PC genes, respectively. Dark green circles represent genes contained in each $\mathrm{CCl}_{4}$ cluster $(\mathbf{c}, \mathbf{d})$. Western blot analysis of whole liver extracts after $\mathrm{CCl}_{4}$ intoxication. Blots are representative from at least three biological replicas (color figure online)

principles were also obtained for other canonical pathways, e.g. "Unfolded protein response" triggered upregulation of the hepatocyte transcription factor CEBPB, while CEBPA and SREBF1 were downregulated (Fig. 4d). Therefore, activation of inflammation (and/or ER stress) and suppression of metabolic genes after $\mathrm{CCl}_{4}$ intoxication may be caused by the same upstream regulators and canonical pathways (Fig. 4b-d).

To study if the observations made in $\mathrm{CCl}_{4}$ intoxicated mice apply also to further models of hepatotoxicity, we analyzed in vivo interventions with LPS (inflammation) and tunicamycin-Tm (ER stress) under conditions where (in contrast to the $\mathrm{CCl}_{4}$ protocol) only little necrosis is induced, using previously reported gene array studies (Supplement 11). A highly significant overlap of downregulated genes in LPS and Tm versus CCl4-treated livers (Fig. 4e) was observed, which was enriched in KEGG pathways and GO terms representing metabolism such as "small molecule catabolic process", "Steroid metabolic process", "Drug metabolism-cytochrome P450", and "PPAR signaling pathway" (Fig. 4f; Supplement 12). These results indicate that gene networks activated by inflammation (LPS) and/or ER stress (Tm) trigger downregulation of genes responsible for metabolic functions in the liver similar to the $\mathrm{CCl}_{4}$ model.

\section{Inflammation-dependent suppression of metabolic networks in chronic liver disease in mice and human}

The presented gene network analysis suggests that inflammation and ER stress may simultaneously induce upregulation of inflammation-associated and downregulation of metabolic gene networks in an injured liver. Noteworthy, during acute injury hepatocytes express markers of embryonic development such as alpha-fetoprotein (Supplement 2). These features are also observed in immature hepatocytes such as hepatoblasts and stem cell-derived hepatocytes (Godoy et al. 2015). Because dedifferentiation toward a more immature phenotype is also a feature of hepatocellular carcinoma-HCC, we assessed whether similar inflammation (and ER stress)-induced suppression of metabolic networks occurs in HCC. For this, we analyzed previously generated data of HCC that was induced in mouse livers by a single injection of diethylnitrosamine (DEN) followed by repeated injections of $\mathrm{CCl}_{4}$ (Liu et al. 2015) (Supplement 13). Spearman correlation analysis of $\mathrm{HCC}$ and liver tissue after $\mathrm{CCl}_{4}$ for all genes showed a high correlation, particularly on days 1 and 2 after $\mathrm{CCl}_{4}$ (Fig. 5a). The strong overlap between the chronic and acute liver injury model was observed for up and downregulated genes (Fig. 5b). To identify possible commonalities in gene regulatory networks in both models, we analyzed HCC and acute injured liver gene expression by IPA (Supplements 14 and 15). The top-scoring canonical pathway for $\mathrm{HCC}$ and acute $\mathrm{CCl}_{4}$ intoxication was "LPS/IL-1 mediated inhibition of RXR function" (Fig. 5c and Supplement 14 ), with highly significant pathways representing inflammation and liver metabolic networks. Upstream regulators and their active/inactive states were also similar for $\mathrm{HCC}$ and acute $\mathrm{CCl}_{4}$ intoxication and known regulators of liver metabolic functions such as ACOX1, TSC2, mir122 SCAP, HNF4, and SREBF1 were strongly suppressed (Fig. 5d; Supplement 15). Activated ER stress pathways were not detected in experimental HCC suggesting that inflammation, rather than ER stress promotes the suppression of metabolic gene networks in this model.

To establish whether the aforementioned upregulation of inflammation/ER stress and concomitant downregulation of metabolism-associated genes also occur in human liver disease; we analyzed pathways and upstream regulators by IPA in previously reported gene array studies on non-alcoholic fatty liver disease (NAFLD) (Moylan et al. 2014), hepatitis B infection (HBV) (Farci et al. 2010), and HCC (Arai et al. 2003) (Fig. 6; Supplements 16 and 17). Similar to the acute injury/HCC situation, the most significant IPA pathways in chronic human liver disease were related to liver metabolism (e.g. 'FXR/PXR activation', 'LXR/RXR activation'), as well as inflammation (e.g. 'coagulation system', 'LPS/IL-1 mediated inhibition of RXR function') (Fig. 6a; Supplement 16). These scores were higher in HBV and HCC compared to NAFLD. The pathways were composed of up and downregulated genes. For example, among the top upregulated genes were cytokines (IL-18, IL-33), while downregulated genes included P450 enzymes (e.g. Cyp7a1, Cyp2c8), transporters (e.g. Slco1b3), and TFs (e.g. CAR) (Fig. 6b, c). Similar to the mouse models, upstream regulator analysis demonstrated a decrease in mature liver function factors such as HNF4, HNF1A, and PXR (Fig. 6d; Supplement 17). Conversely, regulators associated with inflammation (e.g. Interferon alpha, TGFB 1, IL2, and CD44) were highly activated in all human liver disease conditions. Altogether, the analysis revealed an inflammation-associated suppression of metabolic gene networks in acute and chronic liver injury of human and mouse. 
(A)

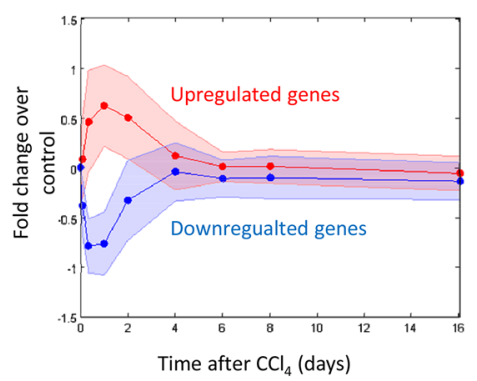

(D)

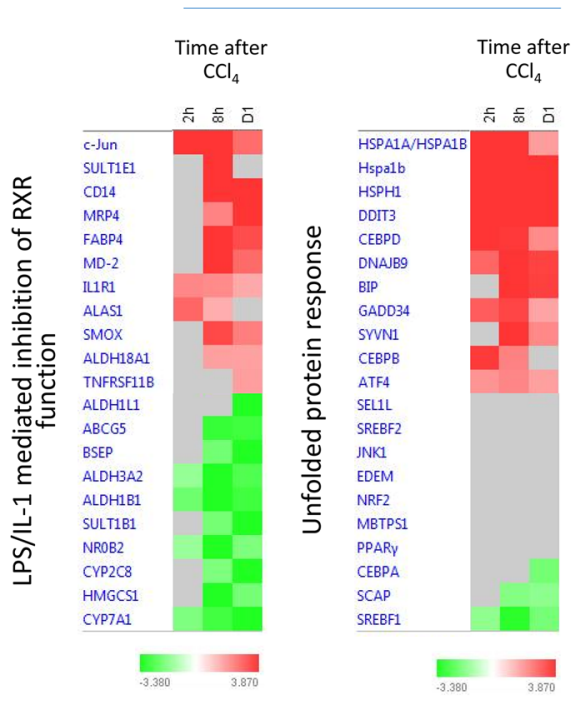

(E)

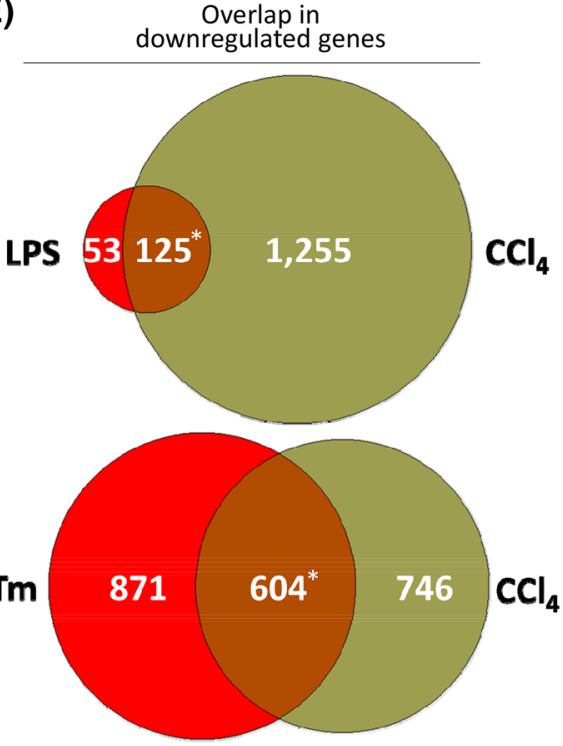

(B)

\begin{tabular}{|c|c|c|c|}
\hline $\begin{array}{c}\text { Canonical } \\
\text { pathways (IPA) }\end{array}$ & 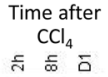 & $\begin{array}{l}\text { Upstream } \\
\text { regulators (IPA) }\end{array}$ & 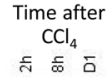 \\
\hline LPS/L-1 Mediated Inhibition of RXR Function & & IL1B & \\
\hline Unfolded protein response & & NUPR1 & \\
\hline NRF2-mediated Oxidative Stress Response & & PDGF BB & \\
\hline Aryl Hydrocarbon Receptor Signaling & & EGFR & \\
\hline Xenobiotic Metabolism Signaling & & ACOX1 & \\
\hline PXR/RXR Activation & & $\mathrm{XBP1}$ & \\
\hline Superpathway of Cholesterol Biosynthesis & & ERK & \\
\hline Cholesterol Biosynthesis I & & TNF & \\
\hline Cholesterol Biosynthesis II (via 24,25-dihydrol... & & IL1A & \\
\hline Cholesterol Biosynthesis III (via Desmosterol) & & CREB1 & \\
\hline Tryptophan Degradation III (Eukaryotic) & & SCAP & \\
\hline Protein Ubiquitination Pathway & & ERN1 & \\
\hline Valine Degradation I & & IL6 & \\
\hline Serotonin Degradation & & $\begin{array}{l}\text { CREM } \\
\text { EGF }\end{array}$ & \\
\hline Superpathway of Melatonin Degradation & & MKNK1 & \\
\hline Intrinsic Prothrombin Activation Pathway & & TGFB1 & \\
\hline Nicotine Degradation II & & P38 MAPK & \\
\hline Histidine Degradation III & & ERK1/2 & \\
\hline Noradrenaline and Adrenaline Degradation & & ATF4 & \\
\hline Glutathione-mediated Detoxification & & SREBF1 & \\
\hline Endoplasmic Reticulum Stress Pathway & & MYDE8 & \\
\hline Dopamine Degradation & & Mek & \\
\hline Hepatic Cholestasis & & SREBF2 & \\
\hline FXR/RXR Activation & & RELA & \\
\hline Ethanol Degradation II & & TLR2 & \\
\hline Tryptophan Degradation X (Mammalian, via ... & & CST5 & \\
\hline Melatonin Degradation I & & $\mathrm{MAP} 2 \mathrm{~K} 1 / 2$ & \\
\hline Nicotine Degradation III & & Alpha catenin & \\
\hline Coagulation System & & ATP7B & \\
\hline LXR/RXR Activation & & EGR1 & \\
\hline Bile Acid Biosynthesis, Neutral Pathway & & $\begin{array}{l}\text { ERBB2 } \\
\text { PKD1 }\end{array}$ & \\
\hline EFF2 Signaling & & $\begin{array}{l}\text { PKD1 } \\
\text { TNFRSF1A }\end{array}$ & \\
\hline Acetone Degradation I (to Methylglyoxal) & & F7 & \\
\hline Superpathway of Methionine Degradation & & IL17A & \\
\hline Estrogen Biosynthesis & & HNF1A & \\
\hline Acute Phase Response Signaling & & IL5 & \\
\hline Aldosterone Signaling in Epithelial Cells & & NFE2L2 2 & \\
\hline Putrescine Degradation III & & CSF2 & \\
\hline Glucocorticoid Receptor Signaling & & TRIB3 & \\
\hline Histamine Degradation & & HNF4A & \\
\hline \multicolumn{2}{|c|}{$-\log$ (p-value) } & \multicolumn{2}{|c|}{ Activation z-score } \\
\hline 0.00 & 10.7 & -6.695 & 6.440 \\
\hline
\end{tabular}

(F)

\begin{tabular}{|c|c|c|}
\hline \multicolumn{3}{|c|}{$\begin{array}{l}\text { GSEA in overlapped } \\
\text { downregulated genes }\end{array}$} \\
\hline GO-BP & $\begin{array}{c}\text { p-value } \\
\left(\mathrm{LPS}_{\mathrm{C} C C I_{4}}\right)\end{array}$ & $\begin{array}{c}\mathrm{p} \text {-value } \\
\left(\mathrm{Tm} / \mathrm{CCl}_{4}\right)\end{array}$ \\
\hline $\begin{array}{l}\text { Small molecule } \\
\text { metabolic process }\end{array}$ & $2.24 \mathrm{E}-32$ & $5.15 E-76$ \\
\hline $\begin{array}{l}\text { Fatty acid } \\
\text { metabolic process }\end{array}$ & $1.03 \mathrm{E}-32$ & $1.98 \mathrm{E}-27$ \\
\hline $\begin{array}{l}\text { Steroid metabolic } \\
\text { process }\end{array}$ & $7.88 \mathrm{E}-11$ & $4.40 \mathrm{E}-20$ \\
\hline $\begin{array}{l}\text { Xenobiotic } \\
\text { metabolic process }\end{array}$ & $6.52 \mathrm{E}-09$ & 4.47E-06 \\
\hline KEGG & $\begin{array}{c}\text { p-value } \\
(\text { LPS/CCI }\end{array}$ & $\begin{array}{c}\text { p-value } \\
\left(\mathrm{Tm} / \mathrm{CCI}_{4}\right)\end{array}$ \\
\hline $\begin{array}{l}\text { Drug metabolism - } \\
\text { cytochrome p450 }\end{array}$ & $2.35 \mathrm{E}-13$ & 4.17E-07 \\
\hline $\begin{array}{l}\text { Fatty acid } \\
\text { metabolism }\end{array}$ & $1.43 \mathrm{E}-12$ & $2.71 \mathrm{E}-12$ \\
\hline $\begin{array}{l}\text { PPAR signaling } \\
\text { pathway }\end{array}$ & $3.88 \mathrm{E}-09$ & $2.23 \mathrm{E}-07$ \\
\hline$A B C$ transporters & $1.23 \mathrm{E}-05$ & $2.53 E-07$ \\
\hline
\end{tabular}

$\left(*=p<10^{-5}\right)$ 
४Fig. 4 Inflammation and ER stress-dependent suppression of metabolism-associated genes. a Graphical representation of all differential genes after $\mathrm{CCl}_{4}$ intoxication. Dots represent mean scaled values of all genes at each time point. Light red and blue areas represent standard deviation. Heatmap representation of the top 40 canonical pathways (b) and upstream regulators (c) significantly altered during $\mathrm{CCl}_{4}$ intoxication. d Heatmap representation of the top 10 up and downregulated genes in the two strongest overrepresented pathways identified by IPA. e Overlap between downregulated genes after LPS or tunicamycin administration and after $\mathrm{CCl}_{4}$ intoxication (i.e. genes in cluster group 3$)$. Both overlaps were highly significant $\left(<10^{-5}\right)$. f Selected overrepresented GO and KEGG terms in the overlapped genes from (e)

\section{Discussion}

Liver damage leads to the activation of numerous signaling and transcriptional regulatory networks whose functions in tissue injury and regeneration are not fully understood (Forbes and Rosenthal 2014). Here, we applied timeresolved transcriptomics of mouse liver after acute intoxication with $\mathrm{CCl}_{4}$ combined with bioinformatics to unravel the sequential processes during early onset of injury and subsequent regeneration. The most significant biological motifs upon $\mathrm{CCl}_{4}$ administration are upregulated gene clusters representing stress signaling (i.e. endoplasmic reticulum stress) and inflammation, which is accompanied by downregulation of genes representing mature liver functions, particularly endogenous, as well as xenobiotic metabolism. This concomitant inflammation/metabolism response peaks between 1 and $24 \mathrm{~h}$ after $\mathrm{CCl}_{4}$ intoxication. In contrast, genes associated with proliferation and tissue restoration peak only $48 \mathrm{~h}$ after intoxication. Analysis of lobular zonation showed that stress signaling is limited to PC hepatocytes; whereas increased expression of inflammation and reduced expression of metabolism-associated genes occur throughout the liver parenchyma. Thus, suppression of metabolic functions occurs not only in PC hepatocytes that will undergo necrosis upon $\mathrm{CCl}_{4}$ exposure, but also in PP hepatocytes that will survive intoxication. All lobular zones respond already within the first two hours to intoxication, although $\mathrm{CCl}_{4}$ is metabolically activated to its toxic metabolites, trichloromethyl and trichloromethylperoxy radicals, only by approximately $40 \%$ of most central hepatocytes of liver lobules, because only these cells express the toxic enzyme cytochrome P450 2E1 (Godoy et al. 2013).

The significance of suppressing mature liver functions during inflammatory conditions is not fully understood. One possibility is that this response is necessary for efficient regeneration upon acute liver injury. In support, suppressing HNF4 expression in adult mouse liver with a tamoxifencontrolled system decreased multiple genes related to metabolic liver functions, while concomitantly increasing expression of proliferation genes, thus linking HNF4 to hepatocyte proliferation (Bonzo et al. 2012). HNF4 downregulation is also observed in hepatocellular carcinoma (Lazarevich et al. 2004, 2010) and its inhibition leads to epithelial-to-mesenchymal transition in mature hepatocytes and hepatoma cell lines (Santangelo et al. 2011), which may also be necessary for re-establishment of tissue architecture after massive injuries (Hoehme et al. 2010). Furthermore, only a relatively small fraction of the metabolic capacity of the normal liver is sufficient for survival. Therefore, inflammation-mediated downregulation of metabolic enzymes and mature liver functions will not lead to major pathophysiological consequences. It may, however, support the regeneration process by transiently focusing more resources on proliferation and re-establishment of a functional microarchitecture.

An interesting observation is that cluster group 2 genes (associated with mature liver functions downregulated after $\mathrm{CCl}_{4}$ ) are enriched in PP hepatocytes. This downregulation overlaps with the upregulation or nuclear accumulation of the inflammatory transcription factor CEBPD, which not only occurs in Cyp2E1-positive PC, but also expands to most PP hepatocytes. Ingenuity pathway analysis provides support that the same upstream regulators are responsible for both the upregulation of inflammatory genes and the downregulation of genes associated with metabolism and mature liver functions. For example, the most enriched IPA pathway 'LPS/IL-1 mediated inhibition of RXR function' comprises upregulation of the pro-inflammatory signaling factor c-Jun and downregulation of the bile salt export carrier BSEP, a typical function of mature livers (Fig. 4d). Similarly, MRP4, a gene known to be upregulated in inflamed and cholestatic livers to export bile acids to blood (Jansen et al. 2017) is increased, while CYP7A1, the gene responsible for a key step in bile acid synthesis is downregulated. Further triggers of the coordinated inflammatory response are cytokines such as TNF, IL5, IL6, and SPP1 and signal transducers, including JUNB, MAPK1, and NFKBIN; however, the underlying mechanisms coupling increased expression of inflammatory genes to reduced expression of genes responsible for mature liver functions remain unknown. Hypotheses include cytokines released into the circulation that influence the entire liver, signals mediated by cell types sensing damage, and releasing inflammatory cytokines such as LSEC (Ding et al. 2010) or cytokines rapidly diffusing from the PC wound to the non-injured PP tissue.

IPA and GSEA showed high overrepresentation of ER stress pathways throughout early stages of acute liver injury. Recently, it was reported that hepatocyte death by $\mathrm{CCl}_{4}$ is mediated by the ER adapter stimulator of interferon genes (STING) via interferon regulatory factor 3 (IRF3) (IrachetaVellve et al. 2016), suggesting that transient ER stress may funnel subsequent inflammatory pathways toward hepatocyte death. However, further studies have revealed protective roles for ER stress signaling in liver pathophysiology. For example, we did not observe a reduction in $\mathrm{CCl}_{4}$-induced 
(A)

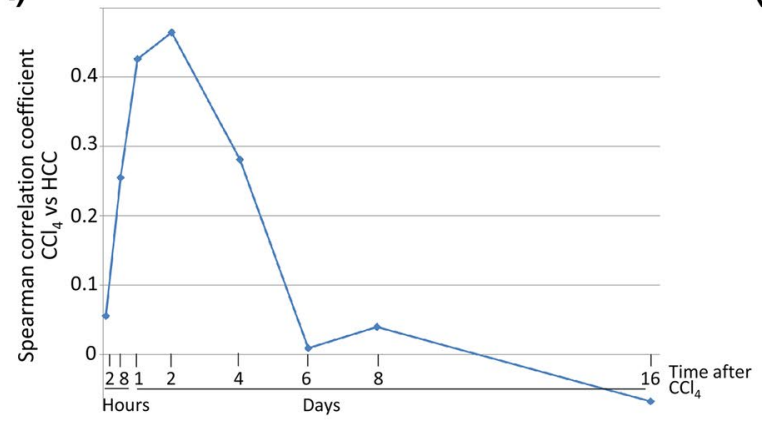

(B)

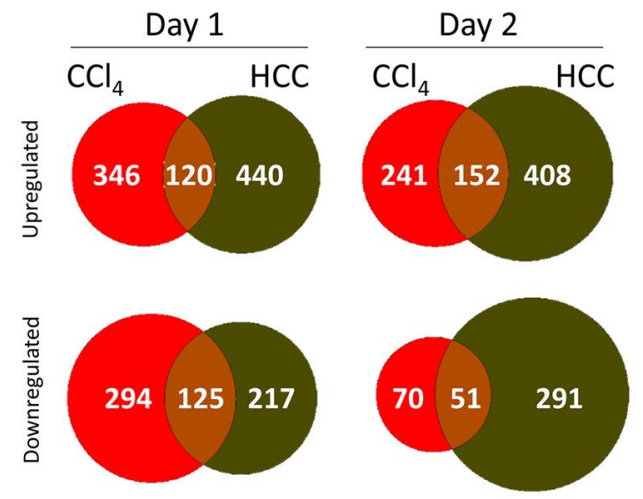

(C)

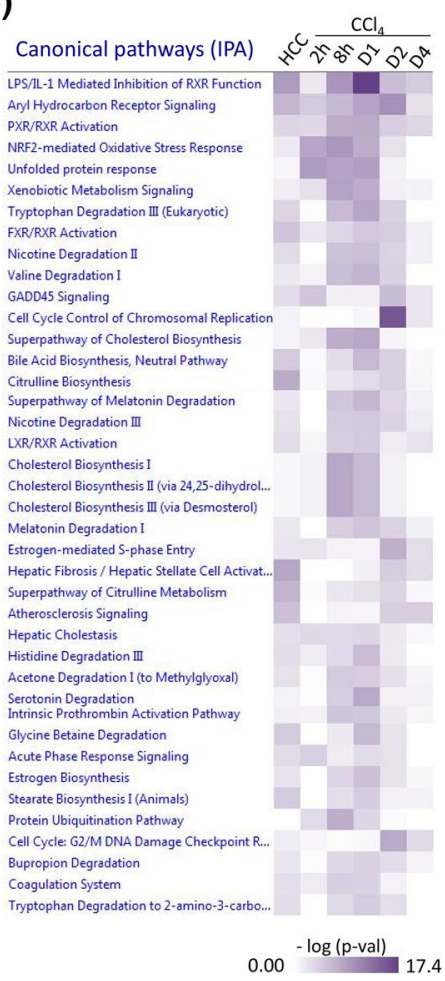

(D)

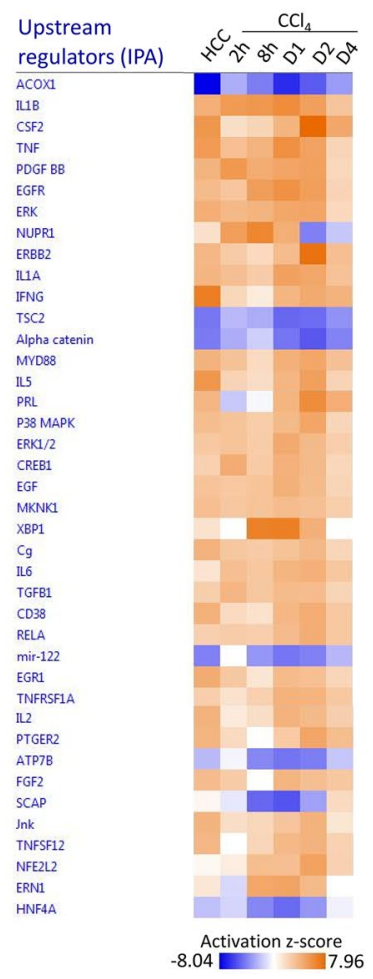

Fig. 5 Identification of inflammation-dependent suppression of liver metabolism gene networks in experimental HCC. a Spearman correlation analysis between differentially expressed genes in HCC vs. acute intoxication with $\mathrm{CCl}_{4}$ (2 $\mathrm{h}$ to day 16$)$. b Venn diagrams showing overlap between upregulated or downregulated genes at the two

liver damage in a knockout mouse model of CHOP, a transcription factor activated in response to ER stress (Campos et al. 2014). Also, hepatocyte-specific deletion of the ER stress sensor, IRE1a, resulted in enhanced liver injury by $\mathrm{CCl}_{4}$, which was associated with dampened STAT3 activation (Liu et al. 2015). Similarly, activated IRE1a protected against acetaminophen hepatotoxicity via the downregulation of Cyp2e1 mRNA due to its RNase activity (Hur et al. 2012). Furthermore, ER stress can affect the functions of non-parenchymal cells, for example, by promoting activation of stellate cells (Kim et al. 2016). Due to this complexity, time points of highest correlation (days 1 and 2) between acute $\mathrm{CCl}_{4}$ intoxication and HCC. All overlaps are highly significant $(p<0.001)$. Heatmap representation of the top 40 canonical pathways (c) or activated/inactivated upstream regulators (d) identified as significantly altered by IPA in $\mathrm{HCC}$ and in acute $\mathrm{CCl}_{4}$ intoxication

the pathophysiological consequences of ER stress are not yet fully understood in the context of liver disease.

The detailed identification of TRNs mediating liver damage and regeneration is fundamental to establish risk, diagnosis, and therapeutic intervention. Our findings provide a detailed, time-resolved description of gene networks in mouse liver upon acute injury and regeneration. Coordinated induction of inflammation/cell stress-associated genes accompanied by downregulation of metabolic genes are controlled by the same upstream regulators and represent a pattern that occurs similarly in acute and chronic liver diseases in humans and mice. 


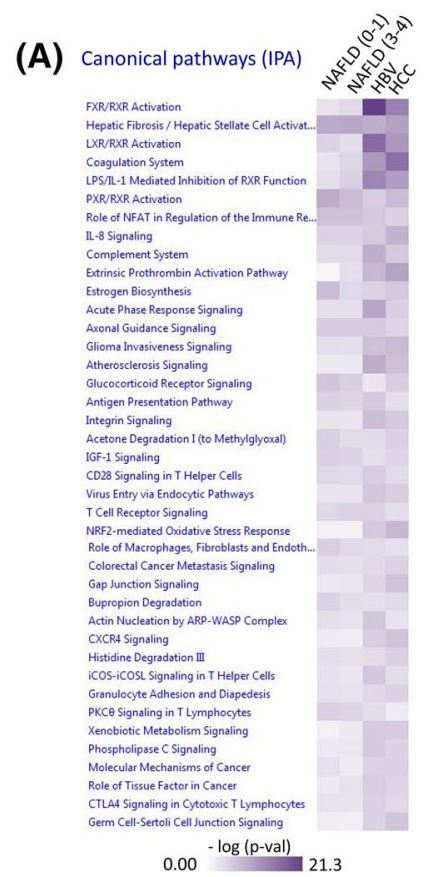

(B)

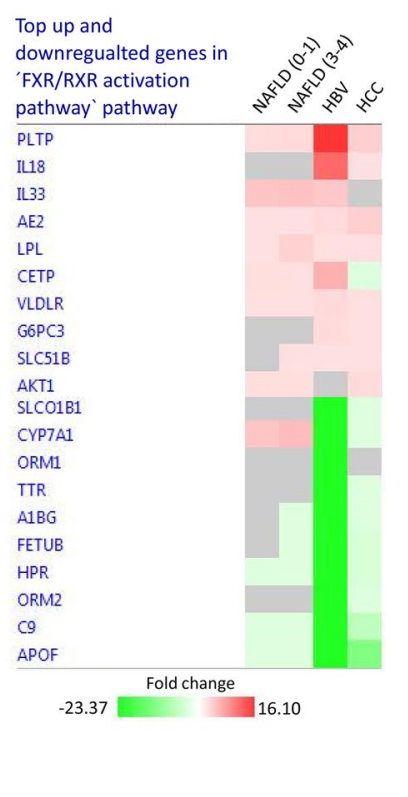

Fig. 6 Identification of an inflammation-dependent suppression of liver metabolism gene networks in human liver disease. a Heatmap representation of the top 30 canonical pathways identified as significantly altered by IPA in non-alcoholic fatty liver disease (score $0-1$; 3-4), hepatitis B infected liver (HBV) and hepatocellular carcinoma

Acknowledgements We thank Ms. Katharina Rochlitz, Ms. Brigitte Begher-Tibbe, and Ms. Georgia Günther (Leibniz Research Centre for Working Environment and Human Factors, Technical University of Dortmund, Germany) for excellent technical assistance. This work has received funding from the European Union's Horizon 2020 research and innovation programme under grant agreement no. 681002 (EU-ToxRisk), from TransQST (No. 116030), LivSysTransfer (BMBF, 031L0119) with contributions from StemCellNet (BMBF, 01EK1604A), LiSyM (BMBF, 031Loo45), and InnoSysTox (BMBF/ EU, 031L0021A) We thank the Permanent Senate Commission on Food Safety (SKLM) for valuable discussion.

Author contributions $\mathrm{GC}, \mathrm{AW}, \mathrm{AG}, \mathrm{LP}$ mouse experiments with $\mathrm{CCl}_{4}$, Tm and LPS challenge; GC, AW, LP western blot analyses; GC histological analysis; WS-H, PG, JS and AS gene array analysis and bioinformatics; AB and MS zonated gene array analysis; $\mathrm{GC}$, DG real-time PCR analysis; GC, JGH and PG drafting of manuscript, acquisition of funding; GC, JGH and PG Study concept and design, critical review of manuscript, study supervision; KE, CC, RM, RR, RG, CV, CH, MS, TSW, JLS, DCH and DD study concept and design, critical revision of manuscript.

Funding This study was supported by the European Union Seventh Framework Programme (FP7) Health projects DETECTIVE (EUproject FP7-Health Grant agreement no. 266838) and NOTOX (EUproject FP7-Health Grant agreement no. 267038) (JGH), the DFG WISP (GO 1987/2-1) (PG), the BMBF (German Federal Ministry of Education and Research) project Virtual Liver (0313854) (JGH), FONDECYT no. 1140549, Millennium Institute no. P09-015-F and FONDAP $15150012(\mathrm{CH})$.

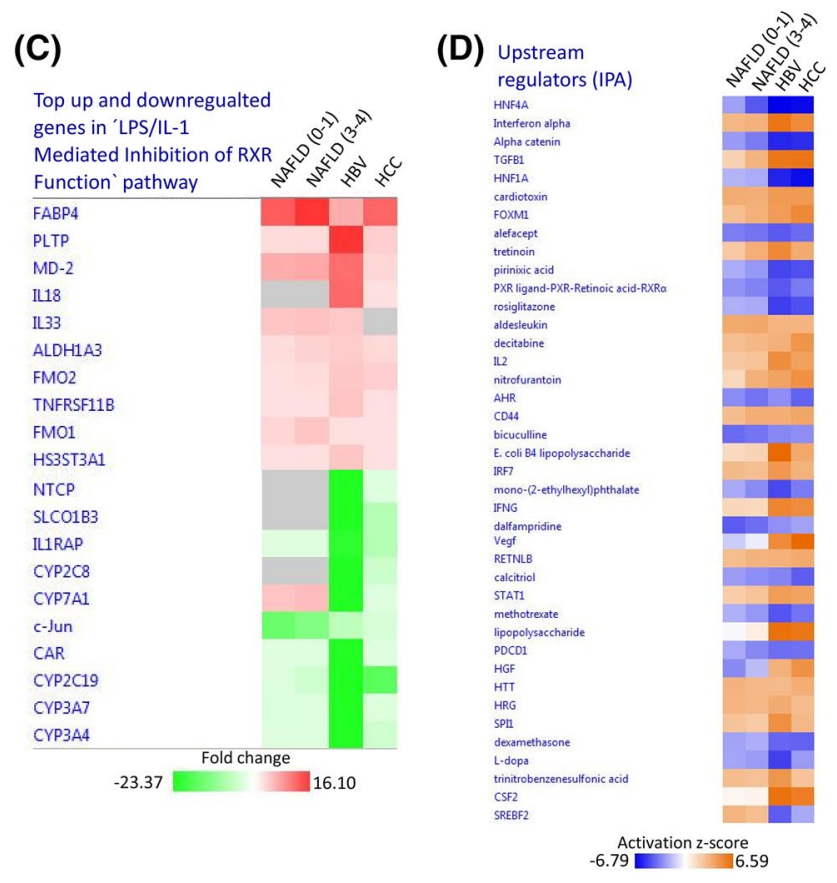

(HCC). b, c Heatmap representation of the top 10 up and downregulated genes in the pathways 'FXR/RXR activation' and 'LPS/IL-1 mediated inhibition of RXR function' identified by IPA. d Heatmap representation of the top 30 upstream regulators identified as significantly activated or deactivated by IPA in human liver disease

\section{Compliance with ethical standards}

Conflict of interest The authors declare that they have no competing interests.

\section{References}

Arai M, Yokosuka O, Chiba T, Imazeki F, Kato M, Hashida J et al (2003) Gene expression profiling reveals the mechanism and pathophysiology of mouse liver regeneration. J Biol Chem 278:29813-29818

Bonzo JA, Ferry CH, Matsubara T, Kim JH, Gonzalez FJ (2012) Suppression of hepatocyte proliferation by hepatocyte nuclear factor 4alpha in adult mice. J Biol Chem 287:7345-7356

Braeuning A, Ittrich C, Kohle C, Hailfinger S, Bonin M, Buchmann A et al (2006) Differential gene expression in periportal and perivenous mouse hepatocytes. FEBS J 273:5051-5061

Campos G, Schmidt-Heck W, Ghallab A, Rochlitz K, Putter L, Medinas DB et al (2014) The transcription factor CHOP, a central component of the transcriptional regulatory network induced upon $\mathrm{CCl} 4$ intoxication in mouse liver, is not a critical mediator of hepatotoxicity. Arch Toxicol 88:1267-1280

Chung H, Hong DP, Jung JY, Kim HJ, Jang KS, Sheen YY et al (2005) Comprehensive analysis of differential gene expression profiles on carbon tetrachloride-induced rat liver injury and regeneration. Toxicol Appl Pharmacol 206:27-42

Ding BS, Nolan DJ, Butler JM, James D, Babazadeh AO, Rosenwaks $\mathrm{Z}$ et al (2010) (2010) Inductive angiocrine signals from 
sinusoidal endothelium are required for liver regeneration. Nature 468:310-315

Dubois-Pot-Schneider H, Fekir K, Coulouarn C, Glaise D, Aninat C, Jarnouen $\mathrm{K}$ et al (2014) Inflammatory cytokines promote the retrodifferentiation of tumor-derived hepatocyte-like cells to progenitor cells. Hepatology 60:2077-2090

Farci P, Diaz G, Chen Z, Govindarajan S, Tice A, Agulto L et al (2010) $B$ cell gene signature with massive intrahepatic production of antibodies to hepatitis B core antigen in hepatitis B virus-associated acute liver failure. Proc Natl Acad Sci USA 107:8766-8771

Forbes SJ, Newsome PN (2016) Liver regeneration-mechanisms and models to clinical application. Nat Rev Gastroenterol Hepatol 13:473-485

Forbes SJ, Rosenthal N (2014) Preparing the ground for tissue regeneration: from mechanism to therapy. Nat Med 20:857-869

Godoy P, Hewitt NJ, Albrecht U et al (2013) Recent advances in 2D and $3 \mathrm{D}$ in vitro systems using primary hepatocytes, alternative hepatocyte sources and non-parenchymal liver cells and their use in investigating mechanisms of hepatotoxicity, cell signaling and ADME. Arch Toxicol 87(8):1315-1530. https://doi.org/10.1007/ s00204-013-1078-5

Godoy P, Schmidt-Heck W, Natarajan K, Lucendo-Villarin B, Szkolnicka D, Asplund A et al (2015) Gene networks and transcription factor motifs defining the differentiation of stem cells into hepatocyte-like cells. J Hepatol 63:934-942

Hart LS, Cunningham JT, Datta T, Dey S, Tameire F, Lehman SL et al (2012) ER stress-mediated autophagy promotes Myc-dependent transformation and tumor growth. J Clin Investig 122:4621-4634

Hoehme S, Brulport M, Bauer A, Bedawy E, Schormann W, Hermes $\mathrm{M}$ et al (2010) Prediction and validation of cell alignment along microvessels as order principle to restore tissue architecture in liver regeneration. Proc Natl Acad Sci USA 107:10371-10376

Hur KY, So JS, Ruda V, Frank-Kamenetsky M, Fitzgerald K, Koteliansky V et al (2012) IRE1alpha activation protects mice against acetaminophen-induced hepatotoxicity. J Exp Med 209:307-318

Iracheta-Vellve A, Petrasek J, Gyongyosi B, Satishchandran A, Lowe P, Kodys K et al (2016) Endoplasmic reticulum stress-induced hepatocellular death pathways mediate liver injury and fibrosis via stimulator of interferon genes. J Biol Chem 291:26794-26805

Jansen PL, Ghallab A, Vartak N, Reif R, Schaap FG, Hampe J, Hengstler JG (2017) The ascending pathophysiology of cholestatic liver disease. Hepatology 65(2):722-738. https://doi.org/10.1002/ hep. 28965

Karin M, Clevers H (2016) Reparative inflammation takes charge of tissue regeneration. Nature 529:307-315

Kim RS, Hasegawa D, Goossens N, Tsuchida T, Athwal V, Sun X et al (2016) The XBP1 arm of the unfolded protein response induces fibrogenic activity in hepatic stellate cells through autophagy. Sci Rep 6:39342

Lazarevich NL, Cheremnova OA, Varga EV, Ovchinnikov DA, Kudrjavtseva EI, Morozova OV et al (2004) Progression of HCC in mice is associated with a downregulation in the expression of hepatocyte nuclear factors. Hepatology 39:1038-1047

Lazarevich NL, Shavochkina DA, Fleishman DI, Kustova IF, Morozova OV, Chuchuev ES et al (2010) Deregulation of hepatocyte nuclear factor 4 (HNF4)as a marker of epithelial tumors progression. Exp Oncol 32:167-171

Liu Y, Shao M, Wu Y, Yan C, Jiang S, Liu J et al (2015) Role for the endoplasmic reticulum stress sensor IRE1alpha in liver regenerative responses. J Hepatol 62:590-598

Michalopoulos GK (2013) Principles of liver regeneration and growth homeostasis. Compr Physiol 3:485-513

Moylan CA, Pang H, Dellinger A, Suzuki A, Garrett ME, Guy CD et al (2014) Hepatic gene expression profiles differentiate presymptomatic patients with mild versus severe nonalcoholic fatty liver disease. Hepatology 59:471-482

Ogata M, Hino S, Saito A, Morikawa K, Kondo S, Kanemoto S et al (2006) Autophagy is activated for cell survival after endoplasmic reticulum stress. Mol Cell Biol 26:9220-9231

Rashid HO, Yadav RK, Kim HR, Chae HJ (2015) ER stress: autophagy induction, inhibition and selection. Autophagy 11:1956-1977

Santangelo L, Marchetti A, Cicchini C, Conigliaro A, Conti B, Mancone $\mathrm{C}$ et al (2011) The stable repression of mesenchymal program is required for hepatocyte identity: a novel role for hepatocyte nuclear factor 4alpha. Hepatology 53:2063-2074

Wang B, Cai SR, Gao C, Sladek FM, Ponder KP (2001) Lipopolysaccharide results in a marked decrease in hepatocyte nuclear factor 4 alpha in rat liver. Hepatology 34:979-989

White P, Brestelli JE, Kaestner KH, Greenbaum LE (2005) Identification of transcriptional networks during liver regeneration. J Biol Chem 280:3715-3722

Young MB, DiSilvestro MR, Sendera TJ, Freund J, Kriete A, Magnuson SR (2003) Analysis of gene expression in carbon tetrachloride-treated rat livers using a novel bioarray technology. Pharmacogenom J 3:41-52

Zidek N, Hellmann J, Kramer PJ, Hewitt PG (2007) Acute hepatotoxicity: a predictive model based on focused illumina microarrays. Toxicol Sci 99:289-302

Publisher's Note Springer Nature remains neutral with regard to jurisdictional claims in published maps and institutional affiliations.

\section{Affiliations}

\section{Gisela Campos ${ }^{1}$. Wolfgang Schmidt-Heck ${ }^{2}$ Jonathan De Smedt ${ }^{3}$. Agata Widera ${ }^{1}$ Ahmed Ghallab ${ }^{1,4}$. Larissa Pütter ${ }^{1}$. Daniela González ${ }^{1} \cdot$ Karolina Edlund $^{1}$. Cristina Cadenas ${ }^{1} \cdot$ Rosemarie Marchan ${ }^{1} \cdot$ Reinhard Guthke $^{2}$. Catherine Verfaillie $^{3}$. Claudio Hetz ${ }^{5,6,7,8} \cdot$ Agapios Sachinidis $^{9} \cdot$ Albert Braeuning $^{10,11}$ - Michael Schwarz ${ }^{10}$. Thomas S. Weiß ${ }^{12}$. Benjamin K. Banhart ${ }^{13}$. Jan Hoek ${ }^{13}$. Rajanikanth Vadigepalli ${ }^{13}$. Jeffrey Willy ${ }^{14}$. James L. Stevens ${ }^{15}$. David C. Hay ${ }^{16}$. Jan G. Hengstler ${ }^{1}$. Patricio Godoy ${ }^{1}$}

1 IfADo-Leibniz Research Centre for Working Environment and Human Factors, Technical University Dortmund, Ardeystrasse 67, 44139 Dortmund, Germany

2 Leibniz Institute for Natural Product Research and Infection Biology e.V., Hans-Knöll Institute, Jena, Germany
3 Stem Cell Institute, KU Leuven, Leuven, Belgium

4 Department of Forensic and Veterinary Toxicology, Faculty of Veterinary Medicine, South Valley University, Qena, Egypt 
5 Biomedical Neuroscience Institute, Faculty of Medicine, University of Chile, Santiago, Chile

6 Center for Geroscience, Brain Health and Metabolism (GERO), Santiago, Chile

7 The Buck Institute for Research in Aging, Novato, CA 94945, USA

8 Department of Immunology and Infectious Diseases, Harvard School of Public Health, Boston, MA, USA

9 Medical Faculty, Institute of Neurophysiology, University of Cologne, Cologne, Germany

10 Department of Experimental and Clinical Pharmacology and Toxicology, University of Tübingen, Tübingen, Germany

11 Department of Food Safety, Federal Institute for Risk Assessment, Max-Dohrn-Str. 8-10, 10589 Berlin, Germany
12 Department of Pediatrics and Juvenile Medicine, Center for Liver Cell Research, University of Regensburg Hospital, Regensburg, Germany

13 Department of Pathology, Anatomy, and Cell Biology, Thomas Jefferson University, Philadelphia, PA, USA

14 Vertex Pharmaceuticals, 3215 Merryfield Row, San Diego, CA 92121, USA

15 Lilly Research Laboratories, Eli Lilly and Company, Lilly Corporate Center, Indianapolis, IN 46285, USA

16 MRC Centre for Regenerative Medicine, University of Edinburgh, Edinburgh E16 4UU, UK 